Revue des patrimoines

46 | 2022

Le patrimoine de la Justice

\title{
Les Baumettes au musée : anciens et nouveaux objets patrimoniaux, de la guillotine aux graffitis
}

The Baumettes at the museum: old and new heritage objects, from the guillotine to graffiti

\section{Zoé Carle et Jean-Fabien Philippy}

\section{(2) OpenEdition}

\section{Journals}

Édition électronique

URL : https://journals.openedition.org/insitu/34052

DOI : 10.4000/insitu.34052

ISSN : $1630-7305$

Éditeur

Ministère de la Culture

Référence électronique

Zoé Carle et Jean-Fabien Philippy, « Les Baumettes au musée : anciens et nouveaux objets

patrimoniaux, de la guillotine aux graffitis », In Situ [En ligne], 46 | 2022, mis en ligne le 16 janvier 2022, consulté le 03 février 2022. URL : http://journals.openedition.org/insitu/34052 ; DOI : https://doi.org/ 10.4000/insitu.34052

Ce document a été généré automatiquement le 3 février 2022

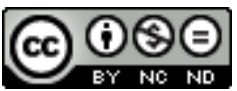

In Situ Revues des patrimoines est mis à disposition selon les termes de la licence Creative Commons Attribution - Pas d'Utilisation Commerciale - Pas de Modification 4.0 International. 


\title{
Les Baumettes au musée : anciens et nouveaux objets patrimoniaux, de la guillotine aux graffitis
}

The Baumettes at the museum: old and new heritage objects, from the guillotine to graffiti

\author{
Zoé Carle et Jean-Fabien Philippy
}

Le musée des civilisations de l'Europe et de la Méditerranée (Mucem) inauguré à Marseille en 2013 est un établissement public national à caractère administratif (EPCA) placé sous la tutelle du ministère de la Culture. Il a notamment pour mission statutaire « de conserver et de présenter au public, en les situant dans leur perspective historique et anthropologique, des biens culturels représentatifs des arts et civilisations de l'Europe et de la Méditerranée ${ }^{1} »$. Le Mucem gère aujourd'hui une collection plurielle et originale dans le monde des musées regroupant des objets d'une grande diversité, pour une large part hérités du musée national des Arts et Traditions populaires (MNATP). Le projet scientifique du MNATP défini dans les années 1930 par Georges Henri Rivière et les chercheurs du musée prêtait une attention particulière aux pratiques et représentations populaires, par la médiation de l'objet, et grâce à l'outil méthodologique des enquêtes-collectes visant à réunir et à documenter des ensembles d'objets tant matériels qu'immatériels, témoins de faits de société : « L'objet ne sera pas considéré comme une simple curiosité ou une valeur purement esthétique mais comme signe matériel de quelque chose de vivant, en l'occurrence les techniques, coutumes, traditions, représentations qui ont cours dans les milieux populaires ${ }^{2}$." Le Mucem s'inscrit explicitement dans la continuité de ce projet, à la fois sur le plan théorique et méthodologique, élargissant le champ des enquêtes-collectes à l'aire méditerranéenne, de façon à constituer des « corpus qui pourront faire l'objet de comparaisons à l'échelle euro-méditerranéenne ${ }^{3}$ ». La perspective d'ethnologie du patrimoine propre à ce musée de société permet une compréhension large de ce qui peut faire patrimoine et c'est particulièrement vrai pour tout ce qui a trait à la prison. 
2 Les collections du Mucem présentent ainsi des traces éparses d'un patrimoine carcéral entendu de façon singulière. Le musée conserve à ce titre deux guillotines ${ }^{4}$, entrées dans les collections publiques quelques mois après l'abolition de la peine de mort en 1981, ainsi que quelques représentations liées à l'univers carcéral jusqu'à la peine capitale : scènes d'exécution en place publique lors de la Révolution française, affaires criminelles ayant défrayé la chronique aux $\mathrm{xIX}^{\mathrm{e}}$ et $\mathrm{xx}^{\mathrm{e}}$ siècles, exécution capitale au bagne de Saint-Laurent-du-Maroni (Guyane). La prison est présente dans les collections sous la forme d'estampes, de caricatures, de cartes postales, d'objets rassemblés au gré de différentes acquisitions, achats et dons, ou d'enquêtes-collectes. C'est ainsi le cas des éléments de l'enquête VIH / sida qui a collecté divers éléments relatifs à la réduction des risques en prison : tracts, flyers, matériel de sensibilisation et de prévention.

En 2019, la question de la prison est relancée au musée avec le déclenchement de l'enquête "Graffitis et créations carcérales ", motivée par la destruction des bâtiments de la prison des Baumettes à Marseille. Celle-ci, construite d'après les plans de Gaston Castel (1886-1971) à partir de 1932, fut inaugurée en 1946 par l'administration pénitentiaire et constitue une "référence incontournable dans l'imaginaire collectif ${ }^{5}$ ». Le centre pénitentiaire des Baumettes est une maison d'arrêt et un centre de semiliberté qui doit sa célébrité autant aux "personnalités » qui y ont séjourné qu'à sa vétusté et à l'insalubrité de ses locaux ${ }^{6}$. Suite à la désaffection des bâtiments dits des «Baumettes historiques ", le Mucem s'est trouvé associé à une mission de conservation d'urgence en collaboration avec le service de l'Inventaire général du patrimoine culturel PACA sur les bâtiments "historiques ", occasion de relire la présence de la prison dans les collections mais également de réfléchir au regard singulier que peut porter un musée de société sur l'univers carcéral à l'époque contemporaine. L'enquête permet ainsi l'extension du domaine d'un "patrimoine carcéral » vers des éléments relatifs au quotidien de l'incarcération, fidèle en ce sens au projet scientifique et culturel du musée.

4 Pourtant, même au sein d'un musée de société, la prison reste un sujet délicat. En témoignent le parcours d'un objet "encombrant» comme la guillotine, et la visibilisation tardive de ces " écritures clandestines ${ }^{7}$ ", les graffitis de détenus. Dans les deux cas, les processus de patrimonialisation sont tortueux et rendent compte à leur façon des difficultés que soulève un patrimoine carcéral suscitant rejet, incompréhension et faisant débat.

\section{La guillotine, objet d'un encombrement patrimonial ?}

Le 17 septembre 1981, Robert Badinter, garde des sceaux et ministre de la Justice, prononce devant l'Assemblée nationale son discours contre la peine de mort. Le 28 septembre, l'abolition de la peine capitale est débattue au Sénat et le 9 octobre, la loi $\mathrm{n}^{\circ}$ 81-908 est promulguée. Le 12 novembre, le comité consultatif des Musées nationaux se réunit et examine le projet d'entrée dans les collections publiques de deux guillotines que la Chancellerie destine au domaine des musées, et qui seront inscrites à l'inventaire du MNATP. La rapidité avec laquelle cette question est traitée pourrait paraître étonnante - quelques semaines seulement séparent la promulgation de la loi et la séance du comité consultatif des Musées nationaux - mais en réalité elle ne l'est pas tant que ça car la décision de patrimonialiser ces objets avait été prise par Robert Badinter. Les sources dont nous disposons pour retracer leur parcours ${ }^{8}$ et l'entrée dans 
les collections publiques sont lacunaires, tenant autant à l'histoire de l'institution qu'à l'objet lui-même, à ce qu'il suscite ou provoque - rejet, dégoût, fascination plus ou moins morbide. Néanmoins, il est cependant possible de définir deux périodes pouvant caractériser son histoire: une période d'invisibilité et une autre, inverse, où la guillotine (re)devient visible, exposée.

\section{La guillotine, objet invisible}

6 L'invisibilité commence paradoxalement dès l'entrée dans les collections publiques. Non pas que celle-ci se soit faite de manière clandestine, puisqu'un procès-verbal est établi lors de chaque réunion du comité, mais du fait du changement de statut de l'objet lui-même et de la restriction qui l'accompagne. En effet, lors du comité consultatif des Musées nationaux, le conservateur en chef du MNATP fait part d'une communication du ministère de la Justice au directeur du musée: «La guillotine, qui n'a plus dorénavant son utilité serait remise au(x) Musées nationaux mais en tout état de cause elle ne devrait pas être exposée avant l'An $2000^{9}$ ». Faut-il voir dans cette restriction le programme d'une disparition de l'objet? Nous sommes à quelques semaines de l'abolition, le sujet reste vif et il faut apaiser le débat, calmer les esprits. L'opinion publique en France est alors plus favorable à la peine capitale qu'à son abolition. En septembre 1981, un sondage Figaro-Sofres montre que $62 \%$ des Français sont pour le maintien de la peine de mort alors que le 30 avril 1980, (seulement) 58 \% des Français y étaient favorables ${ }^{10}$. La restriction apparait donc plutôt comme une mesure de prudence.

7 La guillotine entre dans les collections publiques sans autre publicité. Peu d'objets sont autant sujets à polémiques, controverses, débats, fantasmes. La conservation de ces deux guillotines est très tôt devenue la pierre d'achoppement entre deux institutions, le musée Carnavalet, relevant des musées de la Ville de Paris, et le MNATP, musée national sous la tutelle du ministère de la Culture. Dès la réunion du comité consultatif des Musées nationaux, «Le Directeur précise que $\mathrm{M}^{\mathrm{e}}$ Badinter a fait téléphoner pour annoncer cette nouvelle et que le problème de l'affectation des Bois de Justice s'est posé alors. Le Musée Carnavalet ne s'est pas opposé à leur conservation dans ses collections mais ils seront inscrits à l'inventaire du Musée des Arts et Traditions Populaires ${ }^{11}$ ». Plusieurs courriers sont échangés entre les deux institutions entre 1982 et 1986 concernant cette « conservation dans les collections» qui se traduit dans les faits par une demande de mise en dépôt du MNATP au musée Carnavalet. La première demande doit probablement se faire durant l'année $1982^{12}$.

Le 2 août 1983, Bernard de Montgolfier, inspecteur général des Musées de la Ville de Paris et conservateur en chef du musée Carnavalet, précise dans une lettre adressée à Jean Cuisenier, directeur de recherche au CNRS. et conservateur en chef du MNATP, que "d'importants travaux vont dans un proche avenir rendre pratiquement indisponibles les réserves du Musée Carnavalet » avant d'ajouter : "Ces difficultés ne remettent nullement en cause le principe du dépôt. Je souhaite cependant que le transport effectif des deux guillotines soit différé jusqu'à ce que les réserves du Musée Carnavalet soient en état de les recevoir, le délai étant de trois ans minimum ${ }^{13}$ ». En août 1983, les deux guillotines ne sont pas dans les réserves du MNATP, ni en dépôt au musée Carnavalet, mais toujours à la prison de Fresnes (Val-de-Marne). Le 10 août, Jean Cuisenier fait parvenir au directeur de cette prison un courrier où sont précisés 
plusieurs éléments: les termes d'un entretien téléphonique entre l'un de ses collaborateurs et les services pénitentiaires au sujet du transfert des bois de justice dans les locaux du musée Carnavalet ; le départ en vacances de M. Chevalier, exécuteur des hautes-œuvres, ce qui rend impossible avant le mois de septembre l'inventaire afin de constituer un dossier scientifique sérieux; et l'impossibilité pour le musée Carnavalet d'accueillir le dépôt avant quatre ans, entraînant la recherche pour les pouvoirs publics d'une localisation provisoire ${ }^{14}$. La rapidité avec laquelle le devenir de cet objet avait été traité en 1981 diffère assez largement de la suite des événements. Il faut sans doute voir dans ces échanges l'embarras, tant face à ce que l'objet suscite qu'à l'obligation de donner suite à une décision prise à un autre niveau. Il est alors peu étonnant que l'on ne trouve aucune inscription dans le registre d'inventaire de l'année 1982, bien que les deux guillotines portent un numéro qui les rattache précisément à cette année-là. Le dernier courrier entre le MNATP et le musée Carnavalet date du 21 avril 1986 : Jean Cuisenier renouvelle alors la demande de dépôt.

9 Le Figaro publie le 10 janvier 1985 un article indiquant que la guillotine se trouve « $\mathrm{Au}$ musée des Arts et Traditions ». La journaliste Marie-Guy Baron écrit :

Il a été, un moment, question qu'elle soit donnée à la Ville de Paris, au musée Carnavalet. "Non, nous n'avons qu'une maquette", dément le conservateur de permanence. «Oui, elle est bien là. Mais vous ne la verrez pas, répond celui du musée des Arts et Traditions populaires, depuis 1983, elle demeure enfermée dans ses caisses, en pièces détachées, à l'intérieur d'un entrepôt des Musées nationaux. Elle possède un numéro d'inventaire, que j'ai oublié, et reste inaliénable ${ }^{15}$. 》

10 L'objet n'est pas neutre et les réponses rapportées dans cet article traduisent une certaine gêne, une réticence à communiquer : «non, nous n'avons qu'une maquette » ou « on ne peut pas la voir, elle est enfermée dans des caisses ». Les termes employés sont également révélateurs de la manière dont l'objet est perçu, il est question d'entrepôt et non pas de réserve de musée, il possède un numéro d'inventaire mais on l'a oublié... Le transfert de la prison de Fresnes vers cet «entrepôt des musées nationaux» a donc probablement eu lieu entre septembre et décembre 1983. Néanmoins, l'inventaire des différentes pièces constitutives des guillotines et leur marquage ne seront réalisés que bien plus tard.

11 En 1985, le contexte en France a peu évolué et reste plutôt favorable à la peine de mort. Au cours de la législature 1981-1986, six propositions de loi sont déposées en faveur de son rétablissement, deux au Sénat et quatre à l'Assemblée nationale. Ces propositions de loi interviennent en réaction à l'actualité : chaque fois qu'un crime particulièrement atroce ou révoltant est commis, des élus réclament le rétablissement de la peine capitale $^{16}$. Mais en 1985, il est surtout question de rendre irréversible l'abolition de la peine de mort :

En cet automne 1985, au-delà de toutes les réformes arrachées in extremis, ou de l'achèvement du nouveau Code pénal, ma préoccupation première était de rendre irréversible l'abolition de la peine de mort. [...] La loi du 9 octobre 1981 pouvait être abrogée par une nouvelle majorité à l'Assemblée, avec l'assentiment de la majorité du Sénat revenant sur son vote antérieur ou s'abstenant. [...] En France comme dans tous les États européens abolitionnistes, se vérifiait cette règle : l'évolution de la criminalité sanglante était indifférente à la présence ou à l'absence de la peine de mort dans la législation pénale. Mais cette donnée-là ne suffisait pas. Il fallait du temps pour que l'abolition s'enracine dans la mentalité collective et que les Français s'accoutument à vivre sans la guillotine qui reposait à Paris dans les caves du musée des Arts et Techniques [sic] populaires ${ }^{17}$. 
Robert Badinter publie en 2011 le récit de ses années passées à Chancellerie (1981-1986) et s'attarde notamment à la fin de ce passage sur la guillotine. L'expression « dans les caves " n'est pas très heureuse mais elle trahit sans doute aussi à sa manière l'objet caché, l'objet oublié. L'erreur sur l'intitulé précis du musée pourrait renforcer cette idée de perte ou d'oubli mais elle est sans doute ici fortuite. Il serait également intéressant de s'interroger sur le choix du vocabulaire associé à l'objet: dans l'article du Figaro, la guillotine «demeure»; dans le texte de Robert Badinter, la guillotine " repose ".

Marie-Guy Baron, dans ce même article du Figaro, interroge :

«Pourquoi n'est-elle pas exposée tel un symbole visible par tous de l'irréversabilité [sic] de l'abrogation? "Il faut laisser les passions s'apaiser", me répond-on au ministère de la Justice. Cet apaisement aura trente ans pour venir (durée de la prescription en matière civile). "Elle attirerait trop de monde", précise-t-on au musée $^{18}$.» Prescription en matière civile ou restriction jusqu'en l'an 2000, la guillotine restera finalement invisible jusqu'en 2010.

\section{La guillotine, objet exposé} de Guy Cogeval, président du musée d'Orsay, le 25 janvier 2010, pour l'exposition "Crime et châtiment ${ }^{19}$ ". Le 16 février, la guillotine est emballée au fort d'Écouen (Vald'Oise) où elle avait été stockée avant d'être transportée vers le musée d'Orsay ${ }^{20}$. Pour la période 1986-2010, les sources consultées restent muettes sur la présence des guillotines au fort d'Écouen. Le lieu apparaît cependant sous une autre désignation dans l'article du Figaro : c'est l'« entrepôt des musées nationaux » et il est fort probable qu'elles ne l'aient jamais quitté depuis leur sortie de la prison de Fresnes. Le fort d'Écouen, situé sur un terrain limitrophe du parc du château d'Écouen (musée national de la Renaissance), a été affecté au secrétariat d'État à la Culture - direction des Musées de France-, par arrêté du 17 avril 1975, et sert de réserves pour plusieurs musées nationaux. Le choix de ne pas conserver ces objets dans les réserves mêmes du MNATP reste obscur. Les documents conservés dans le dossier d'œuvre ne donnent aucune explication, et l'on ne peut qu'émettre des suppositions : que ni le MNATP ni le musée Carnavalet n'aient voulu de ces objets à la charge historique et symbolique particulièrement forte, et que faute de pouvoir les accueillir au musée ou de les déposer, il a été décidé de les entreposer là où cela était possible, au fort d'Écouen. Mais il n'est peut-être pas si étonnant de les retrouver là-bas car le MNATP a toujours utilisé plusieurs lieux pour entreposer ses collections.

Dans l'exposition du musée d'Orsay, la guillotine, d'une certaine manière, ne sera pas complètement visible [fig. 1]. Elle est presque entièrement recouverte d'un voile noir. Présenté au public pour la première fois, l'objet impressionne par ce qu'il est et ce qu'il représente. La place de la guillotine dans le propos de l'exposition, sa localisation au bout d'une galerie dans un espace assez sombre, les dispositifs scénographiques qui la théâtralisent et l'esthétisent, tous ces choix curatoriaux et de présentation changent sa nature en instaurant une mise à distance. La fonction même de l'objet, son utilité propre n'a plus lieu d'être: la guillotine devient le symbole d'une époque passée et révolue, un objet de l'histoire et non plus de la réalité judiciaire. L'inventaire précis des 
différentes pièces et leur marquage ont probablement été effectués en 2010, dans le contexte de l'exposition.

Figure 1

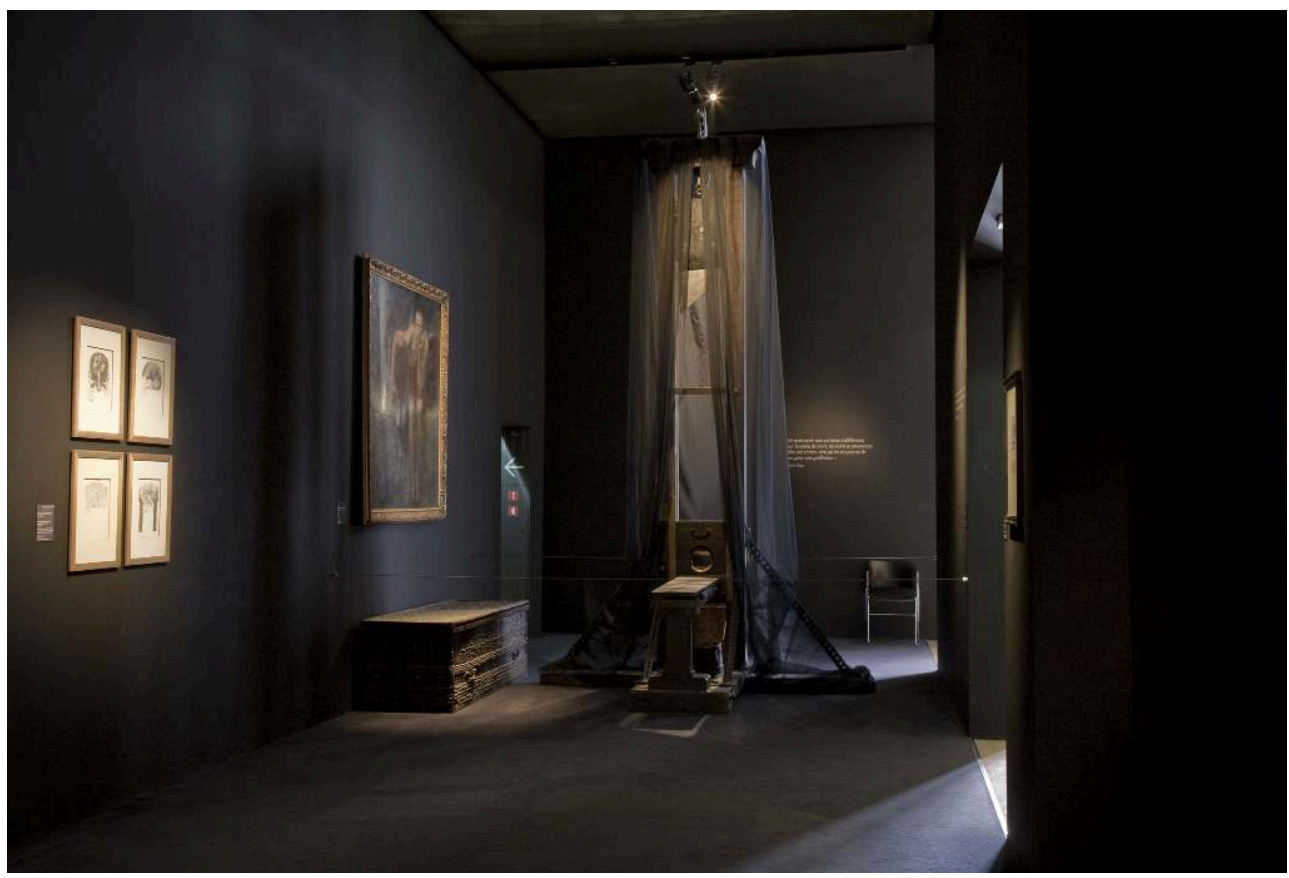

Exposition «Crime et châtiment », Musée d'Orsay, 2010.

(c) Sophie Boegly / reproduction Musée d'Orsay.

16 Par la suite, la guillotine sera présentée à deux reprises : au Mucem, de juin 2013 à novembre 2017, dans le cadre de l'exposition inaugurale «La Galerie de la Méditerranée » dans la section Citoyennetés et droits de l'homme, et aux Baumettes, centre pénitentiaire de Marseille, à titre de prêt exceptionnel, du 18 septembre au 30 novembre 2019. En décalage avec le concept de citoyenneté, la présence de la guillotine dans cette section de la galerie de la Méditerranée est à mettre en lien avec la question des droits de l'homme et à rapprocher de ce que représente l'abolition de la peine de mort : « un progrès de la conscience humaine ${ }^{21}$ ». Le parti-pris scénographique d'un espace sans cimaises avec de grands voiles blancs pour toute structure de parcours n'appartient pas à cette seule présentation mais au projet muséographique d'ensemble. La guillotine se découvre sans autre artifice, au détour du parcours de visite. 
Figure 2

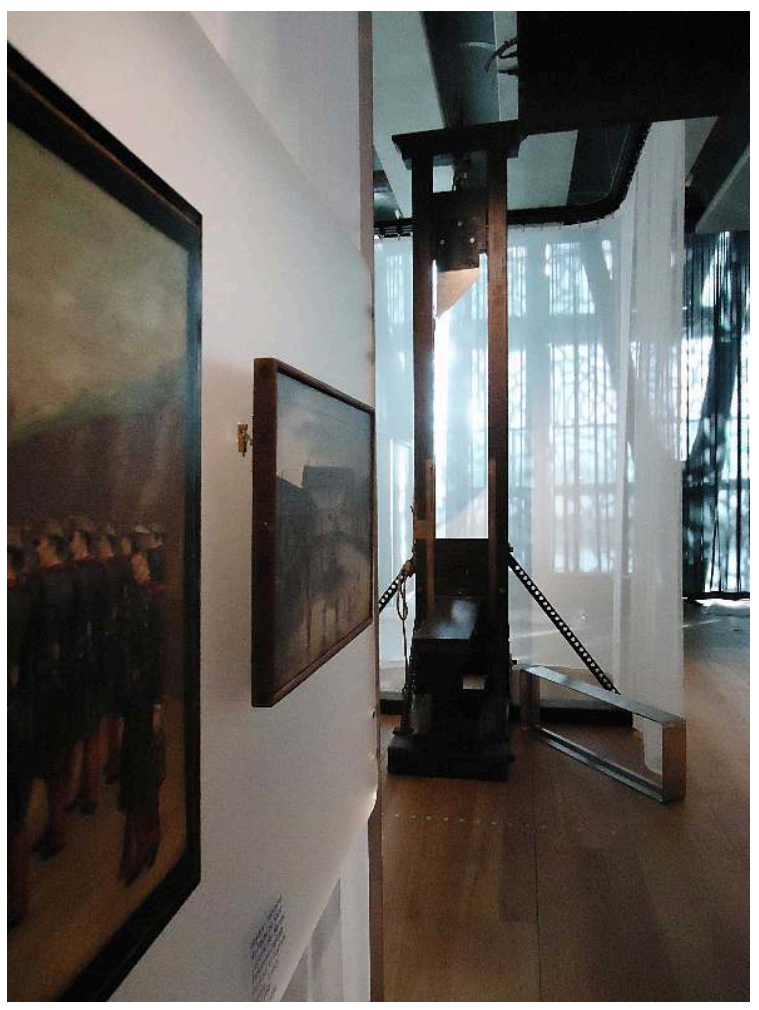

Exposition «La Galerie de la Méditerranée », Mucem, 2013.

(c) Emily Picand / reproduction Mucem.

17 Présentée au Mucem dans un contexte muséal [fig. 2], il en va évidemment tout autrement en ce qui concerne le centre pénitentiaire de Marseille. Les Baumettes dites historiques ferment définitivement en juin 2018 avant une démolition prévue pour 2020-2021. Pierre Raffin, directeur chargé de mission auprès du directeur interrégional des services pénitentiaires de Marseille, et le comité de pilotage de la manifestation «Adieu Baumettes » imaginent alors un projet culturel pour rendre les Baumettes aux Marseillais en rouvrant quelques semaines le site historique et en proposant des visites accompagnées d'une programmation culturelle. Dans ce cadre, l'administration pénitentiaire sollicite le prêt de la guillotine conservée dans les collections du Mucem [fig. 3 et fig. 4]. En inscrivant au sein du projet l'exposition de cet objet - sans scénographie particulière, dans le seul espace pouvant l'accueillir, derrière des barreaux, dans un couloir reliant les bâtiments A et B - la volonté et le choix sont manifestes de ne pas fermer les yeux sur l'histoire de ce lieu puisque la dernière exécution capitale en France avant l'abolition de la peine de mort a eu lieu aux Baumettes, en 1977. La patrimonialisation d'un tel objet ne va peut-être pas de soi, et soulève beaucoup de questions, y compris jusque dans ses formes de valorisation, notamment par l'exposition. Et si l'imaginaire qui l'accompagne reste très prégnant, l'objet semble néanmoins, depuis quelques années, faire moins débat ou polémique au sein de l'institution et sans doute aussi auprès d'une partie du public. 
Figure 3

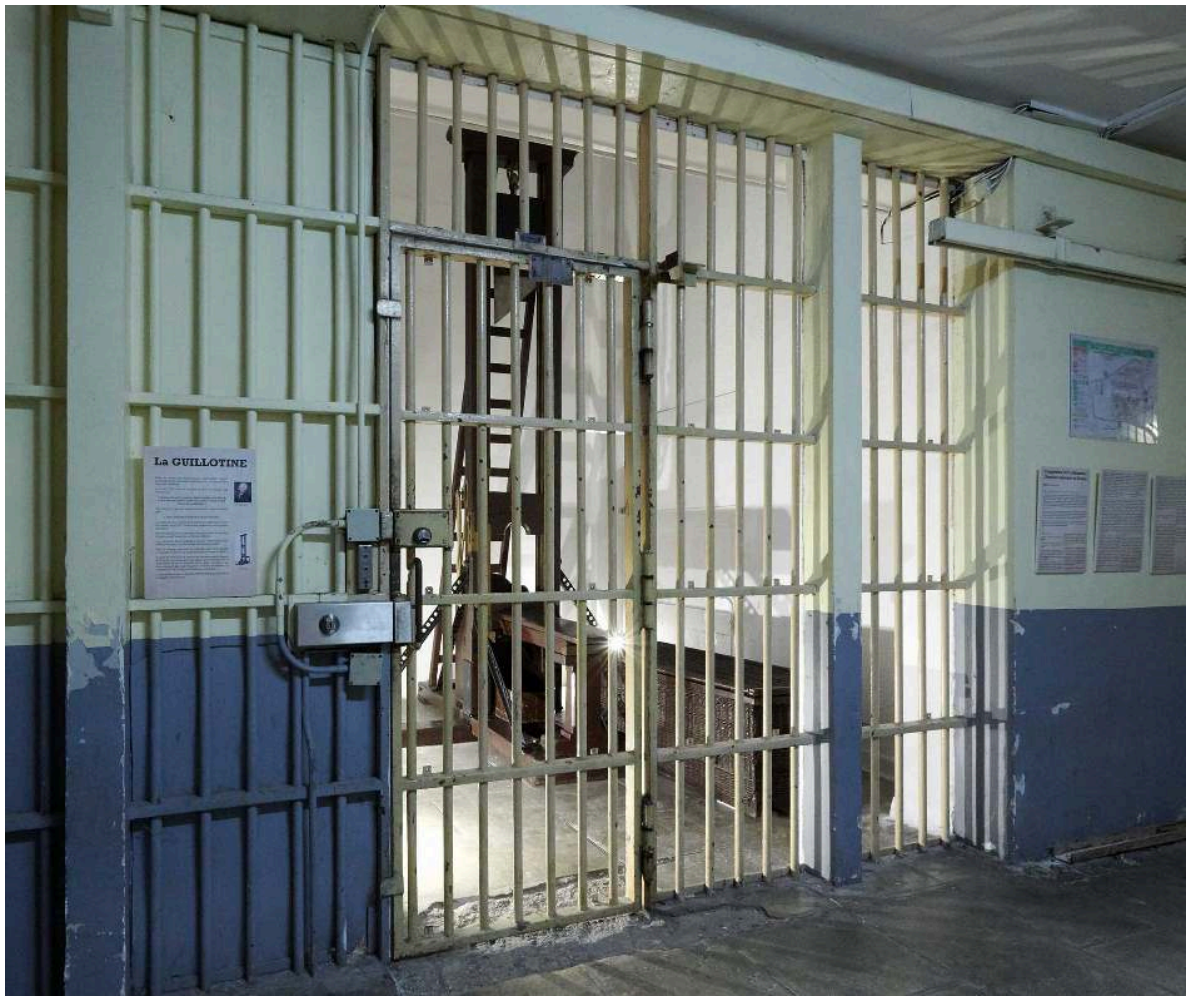

Guillotine, Les Baumettes, centre pénitentiaire de Marseille, 2019.

(c) Marianne Kuhn (Mucem).

Figure 4

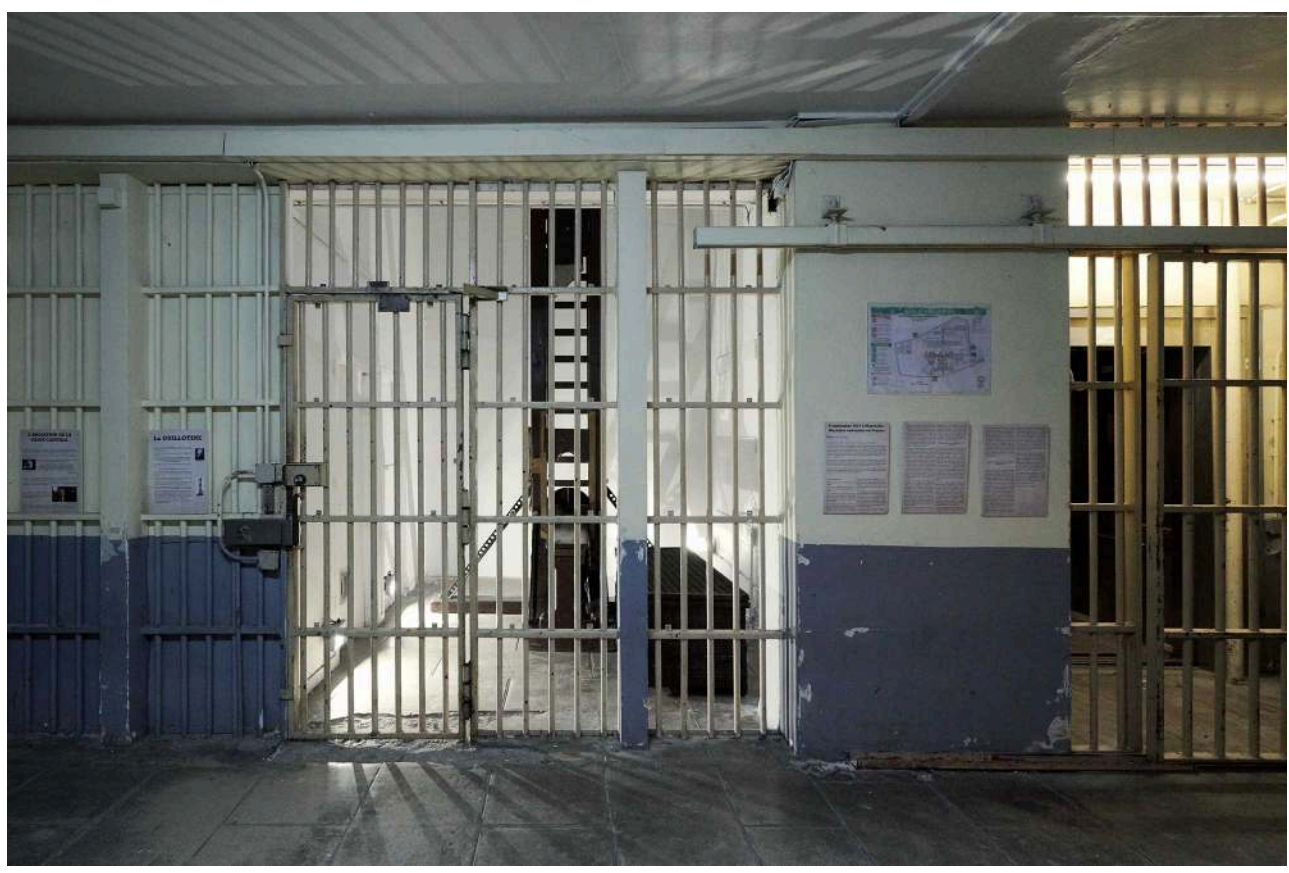

Guillotine, Les Baumettes, centre pénitentiaire de Marseille, 2019.

(C) Marianne Kuhn (Mucem). 
18 Parallèlement à cette manifestation, le Mucem s'est engagé depuis juin 2019, en collaboration avec le service de l'Inventaire général du patrimoine culturel PACA, dans une enquête-collecte prenant spécifiquement les graffitis carcéraux comme objet. Le musée continue ainsi à s'inscrire dans la lignée du MNATP en poursuivant la réalisation d'enquêtes ethnographiques afin de documenter des faits de société contemporains, enquêtes pouvant être entreprises dans le cadre d'opérations de sauvegarde d'un patrimoine ou trouver leur origine dans un fait d'actualité. Ainsi, de façon fortuite, c'est la désaffection d'une partie de la prison des Baumettes qui est à l'origine à la fois d'une nouvelle exposition de la guillotine et d'une enquête-collecte sur un pan longtemps négligé de l'univers carcéral : les graffitis.

\section{Enquête « Graffitis et créations carcérales »}

La notion de patrimoine carcéral vise avant tout le patrimoine mobilier et immobilier de bâtiments vétustes, souvent dégradés ${ }^{22}$, promis à la reconversion ou à la destruction $^{23}$. Elle englobe plus rarement les graffitis de détenus. Du point de vue de l'administration pénitentiaire, ces écrits fragiles, réalisés à la va-vite avec les moyens $\mathrm{du}$ bord sont, au pire, considérés comme des dégradations du bâti, des traces à effacer lorsqu'il s'agit d'ouvrir les lieux au public ${ }^{24}$, au mieux, des inscriptions anodines, sans intérêt réel ${ }^{25}$. Ces derniers se situent ainsi aux antipodes d'un objet aussi visible que la guillotine, qui fait incontestablement partie d'un patrimoine à conserver, même si on n'a pas trop su qu'en faire dans un premier temps.

20 En tant que musée de société, le Mucem semble particulièrement à même d'accueillir un « objet » comme les graffitis, qui se situe à la jonction des productions de justiciables repérables dans les collections, et du continent des "écritures exposées ", modalité singulière des "écritures ordinaires", au statut mi-privé mi-public ${ }^{26}$. De fait, la présence des éléments relatifs à la prison dans les collections se fait par le biais d'une culture populaire attentive à une mythologie du crime et du fait divers ou bien, dans le cas des caricatures du bagne, par des dons de collections privées, en l'occurrence celle d'un administrateur pénitentiaire ayant collecté à titre personnel des productions de bagnards ${ }^{27}$. Paradoxalement, c'est moins la présence d'un patrimoine carcéral disparate que celle des écritures exposées dans les collections qui ouvre la voie à la patrimonialisation des graffitis carcéraux dans le cadre de l'enquête menée sur les Baumettes historiques. Ces derniers sont en effet le point d'entrée de l'enquête, tandis qu'au fur et à mesure seront ajoutés des éléments ayant trait à l'aménagement de l'espace de la cellule par les détenus - affiches, calendriers... - et plus généralement, à l'amélioration des conditions de détention - réchauds, yoyos, rétroviseurs, plateaux d'échecs ... C'est à partir de ce «texte caché » de la prison pourtant que l'enquête démarre, en lien avec les collections d'un musée où la question des écritures exposées ${ }^{28}$, plus spécifiquement des écritures urbaines, a une certaine place.

21 La présence des graffitis est relativement discrète dans les collections du MNATP avant la grande enquête sur le mouvement graff menée par l'ethnologue Claire Calogirou à partir de 2001. Ils sont présents de façon souvent fortuite, à la faveur d'enquêtes ne les prenant pas explicitement comme objet: ainsi les graffitis en milieu rural lors de l'enquête de Jean Cuisenier sur la Basse-Normandie en 1985. Ils font plus rarement l'objet d'enquêtes spécifiques : c'est le cas d'une campagne photographique menée sur le référendum constitutionnel du 5 mai 1946 centrée sur les inscriptions à la craie 
«OUI », « NON » sur les murs de Paris et d'Île-de-France qui comprend également des inscriptions résiduelles de la Seconde Guerre mondiale ${ }^{29}$; mais également de l'enquête photographique menée par Pierre Soulier sur le travail du dessinateur de trottoir Robert Eberlé entre 1945 et 1956, bien avant l'invention du street-art et de ses outils puisque ce dernier réalisait ses dessins à la craie à même le sol.

De façon significative, la présence des écritures exposées s'accélère avec l'entrée dans les collections de l'enquête « Tag et graff » menée à partir de 2002 par Claire Calogirou dans le prolongement de ses travaux précédents sur les pratiques des jeunes en milieu urbain (skate, hip-hop). Les graffitis font donc leur entrée " officielle » au musée par le mouvement graff, qui ne constitue au demeurant qu'une infime portion de la masse des écritures exposées en milieu urbain, mais la plus visible à bien des égards, visibilité que l'on peut imputer à un changement de statut dans les représentations collectives. C'est en effet lorsque ces pratiques scripturaires sont associées à un mouvement artistique protéiforme, le mouvement tag et graff, que les écritures exposées bénéficient d'un regain d'intérêt. La présence des graffitis s'étoffe en effet de façon continue depuis cette enquête, à la fois grâce à des dons personnels - le fonds Polge, consacré au graffiti érotique en 2004, le fonds Cayrol en 2018 - ou par des enquêtes spécifiques qui prennent pour objet les graffitis contestataires depuis $2018^{30}$.

C'est dans ce contexte que s'inscrit l'enquête-collecte "Graffitis et créations carcérales ", débutée en avril 2019, qui élargit encore l'empan de ces écritures ordinaires exposées. Sont concernés à la fois le patrimoine architectural et les graffitis de cellule, qui font tous deux l'objet d'une enquête photographique, éléments complétés par une collecte d'objets du quotidien [fig. 5 et fig. 6]. L'enquête est en ce sens fidèle à la mission qui a été longtemps celle du musée et de la discipline d'ethnologie du patrimoine, cherchant à recueillir les traces de mondes crépusculaires, sur le point de disparaître ${ }^{31}$. Alors que le bâtiment dit des Baumettes historiques est promis à la destruction, la question se pose : que faire de ces traces et écrits? Si l'Inventaire du patrimoine se concentre plutôt sur le bâti, la perspective de l'enquêtecollecte du Mucem est d'aborder la prison par l'autre bout : l'investissement subjectif de l'espace et du temps de la détention, dont les graffitis et les objets fabriqués sont un symptôme et une trace. Il s'agit dès lors de faire place à ce que Philippe Artières nomme "patrimoine noir" des prisons, en d'autres termes, de patrimonialiser des éléments donnant à voir « les instants de subjectivation, à savoir lorsqu'un individu pris dans les mailles du pouvoir se débat avec lui en s'inventant autrement, en se produisant comme sujet $^{32}$ ». Ce faisant, l'enquête rencontre un intérêt récent mais non démenti pour les graffitis carcéraux, longtemps négligés à la fois par les acteurs patrimoniaux et par les milieux scientifiques. 
Figure 5

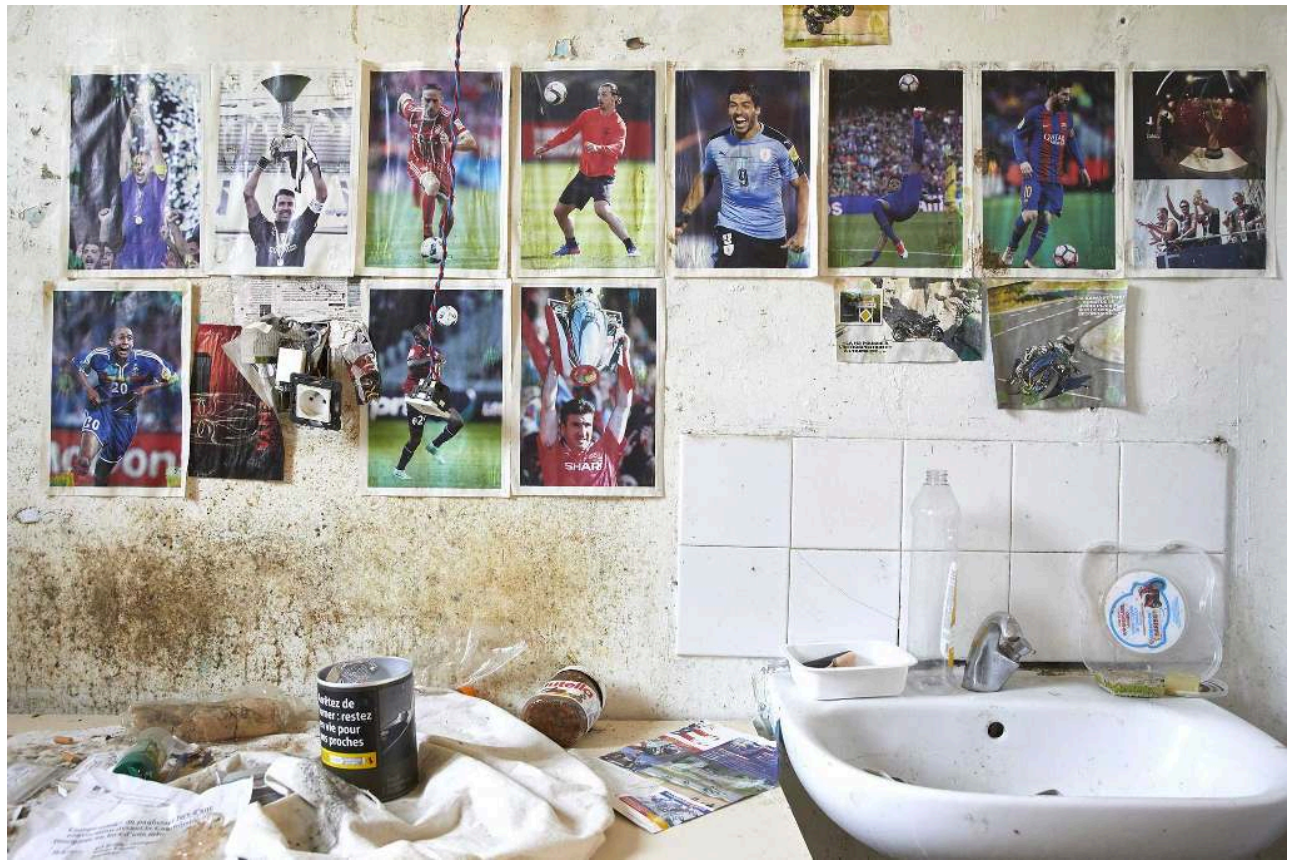

Intérieur d'une cellule, Les Baumettes, centre pénitentiaire de Marseille, 2019.

(c) Frédéric Pauvarel (Inventaire du patrimoine).

Figure 6

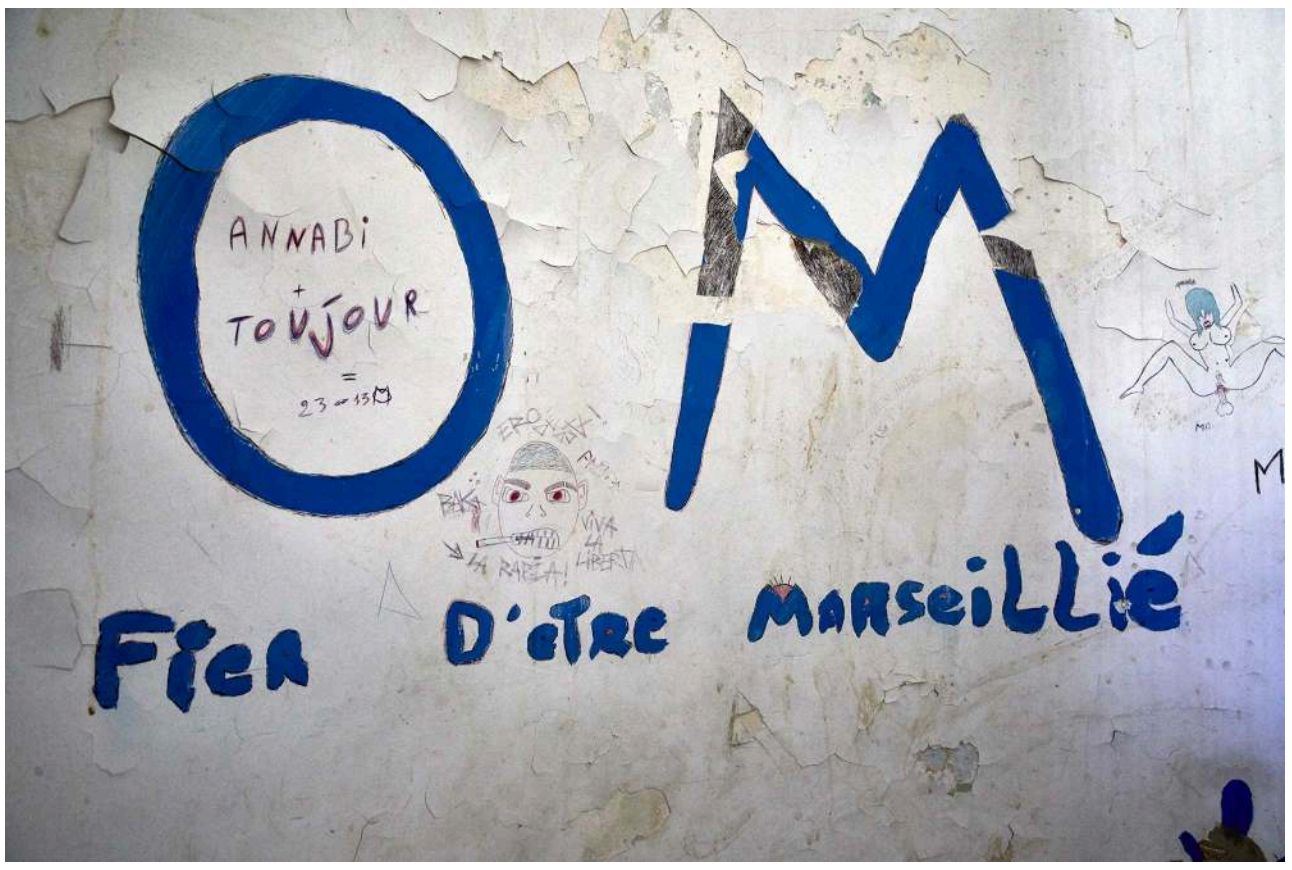

Graffiti de cellule, Les Baumettes, centre pénitentiaire de Marseille, 2019.

(c) Frédéric Pauvarel (Inventaire du patrimoine). 


\section{La longue reconnaissance des graffitis carcéraux}

24 Comme le déplorait Jean-Claude Vimont en 2008, évoquant les "graffitis en péril », au début des années 2000 ces derniers ne faisaient que rarement l'objet d'entreprises de conservation. Depuis, ils font l'objet d'une attention croissante, sous l'impulsion de travaux de recherche qui se sont multipliés à partir des années 1990 sur la question, et comme en témoigne la liste d'initiatives autour des graffitis carcéraux compilées sur le site Criminocorpus ${ }^{33}$. La visibilisation de ces " écritures clandestines» et leur entrée dans la « fabrique du patrimoine ${ }^{34} »$ est directement liée à la valeur, tantôt scientifique, tantôt esthétique qui leur est affectée.

Si les graffitis ont été étonnamment négligés par les acteurs patrimoniaux, ils l'ont été aussi longtemps par la communauté scientifique, comme cela a été remarquablement documenté ${ }^{35}$. Comme le rappelle Philippe Hameau, en dépit même de la prégnance de la thématique de l'enfermement et de l'incarcération en anthropologie, les graffitis n'ont été considérés comme une source qu'à partir des années 1990 en anthropologie, études littéraires et histoire ${ }^{36}$. Cette attention relativement récente doit certainement être mise en rapport avec l'intérêt contemporain pour les «écritures ordinaires ${ }^{37}$ » et plus généralement, pour l'écrit, que ce soit dans une perspective historique ou d'anthropologie de l'écriture, et enfin dans le champ patrimonial ${ }^{38}$.

Les raisons de l'entrée tardive des graffitis carcéraux dans les champs scientifiques puis patrimoniaux sont multiples, comme l'a exposé Anne Monjaret, et apparaît plutôt comme une résurgence lointaine, après un long siècle de désintérêt relatif ${ }^{39}$. Les graffitis de détenus ont en effet suscité un certain intérêt à la fin du xix ${ }^{e}$ siècle, du côté d'une science pénale balbutiante, la criminologie, et sa «clinique de l'écriture ${ }^{40}$ ». Aux côtés des graffitis, les tatouages et plus largement les productions de justiciables chansons, argot, dessins...- ont intéressé des médecins comme Cesare Lombroso (1835-1909), Alexandre Lacassagne (1843-1924) ou Charles Perrier (1862-1938), curieux de percer à jour l'âme de détenus comme ils arpentent leurs corps pour y trouver les traces et les causes du crime car " [1]e délinquant devient individu à connaître ${ }^{41}$ ». De cette «exigence de savoir» découle le "panoptique graphique " décrit par Artières : « la prison va créer de toutes pièces un scripteur et avec lui tout un corpus. Ce scripteur sera le délinquant, ce corpus, la littérature des prisons ${ }^{42}$." L'existence de "codes » propres au genre du graffiti carcéral - les trois points de "mort aux vaches", les cinq points de «seul entre quatre murs » pour ne citer qu'eux - accrédite cette perception d'un langage secret, caché dans les entrailles de la prison et qu'il revient au médecin d'élucider pour accéder à des mondes sociaux et moraux inconnus ${ }^{43}$.

Pourtant, tandis que la science pénale s'éloignait des hypothèses du "criminel-né " d'un Cesare Lombroso ${ }^{44}$, l'intérêt pour les graffitis en tant que source pour l'anthropologie criminelle semble s'être étiolé et ces derniers n'ont pas fait l'objet de collectes systématiques à des fins scientifiques avant le regain d'intérêt des années 1990. Il faut bien entendu mentionner quelques collectes éparses, comme celles d'Henri Calet (1904-1956) sur les graffitis de prisonniers pendant l'Occupation ${ }^{45}$ ou celles de Leonardo Sciascia éditant les graffitis de prisonniers de l'Inquisition compilés par le folkloriste italien Giuseppe Pitrè ${ }^{46}$ (1841-1916) mais ces entreprises s'inscrivent déjà dans une autre « exigence de savoir ». À partir du milieu du xx siècle, les écrits de prisonniers intéressent moins les médecins de prison que des auteurs ou des historiens, sensibles à ces écrits ordinaires, attentifs à une certaine écriture de l'histoire, tandis 
que la valeur qui leur est affectée procède plus du témoignage que de la trace ou de la preuve. Dès lors, les graffitis intéressent parce qu'ils sont produits par des protagonistes plus ou moins anonymes, remarquables, d'une histoire plus ou moins lointaine, dont le récit semble déjà partiellement enclos.

Cela ouvre la voie à la reconnaissance de ces écrits comme source possible d'une écriture de l'histoire, même si, dans le processus de patrimonialisation, la valeur de ces écrits penche désormais moins du côté scientifique que du côté esthétique et émotionnel. De façon paradoxale, les graffitis sont alors rapprochés d'une forme d'art, comme en témoigne l'analyse de Daniel Fabre :

la condition carcérale ne suffit pas à qualifier de façon stable un "art", en revanche, comme elle aboutit à un marquage dense des lieux par les prisonniers eux-mêmes, elle produit un monde de signes forcément obscurs, plus ou moins codés et toujours liés à une situation pathétique que l'histoire magnifie. Cette triple alliance de l'énigme, de la souffrance et du passé contribuera fortement à la pleine conversion du palimpseste des prisons en bien commun, en patrimoine, source d'émotion, d'émerveillement, de dépaysement et d'identification ${ }^{47}$.

Ces inscriptions sont alors dignes d'intérêt en tant $q^{\prime}$ ' $\operatorname{archives~sensibles~}^{48}$ »: non seulement elles permettent un accès au savoir, puisqu'elles sont une source possible de l'écriture de l'histoire, mais elles sont également les traces de la condition douloureuse de l'enfermement et des émotions individuelles que cette dernière suscite.

La mobilisation de la dimension esthétique et pathétique, fondamentale dans la «fabrique du patrimoine » comme l'analysait Nathalie Heinich, nous semble toutefois être liée à un effet de réception contemporaine, qui affecte par contrecoup le statut de ces écrits. Il est probable que l'art contemporain et la photographie en particulier aient joué un rôle important dans la réception de ces écrits jusque-là négligés, comme il n'est pas indifférent que les graffitis aient joui d'un regain de visibilité dans les collections du Mucem grâce au mouvement graff. Immanquablement, lorsque l'on aborde les graffitis, sont évoquées les photographies de Brassaï (1899-1984) et plus spécifiquement dès lors qu'il s'agit de graffitis carcéraux, les travaux de photographes prenant la prison pour objet. Les travaux de Mathieu Pernot (né en 1970), Arnaud Théval (né en 1971), Grégoire Korganow (né en 1967), pour ne citer qu'eux, ont contribué à rendre visibles ces écrits. Visibles mais peut-être pas lisibles, car tel est le paradoxe des graffitis de prison.

31 S'il est en effet commun d'évoquer le statut ambigu des graffitis de prison, entre art et archive, la question artistique ne va pourtant pas de soi. Il s'agit de corpus essentiellement écrits, qui frappent par la monotonie de leurs contenus et thématiques, qui renvoient à la condition d'incarcération de scripteurs n'ayant pas forcément à cœur d'esthétiser leur expérience. Pour le dire autrement, le texte est rarement lu, et les écrits sont traités comme de l'image car leur intérêt est ailleurs. La dimension artistique est certainement à chercher entre cette iconisation de l'écrit et esthétisation d'un univers carcéral qui emprunte aussi ses codes à une esthétique des ruines contemporaines ${ }^{49}$. De fait, les graffitis s'intègrent parfaitement à la thématisation de l'insalubrité et la vétusté des locaux - et leurs conséquences très matérielles sur la vie quotidienne des détenus - et participent ainsi à la constitution d'une certaine esthétique, celle du genre de la "photo de prison», qui a un effet rebond sur une « valeur » patrimoniale ambiguë, à manipuler avec précaution. 


\section{Guillotine et graffitis, le patrimoine négatif de la prison?}

Nathalie Heinich rappelle l'importance des émotions dans la fabrique patrimoniale. Or, les émotions que font naître aussi bien la guillotine que les graffitis de détenus sont incontestablement des émotions négatives. En d'autres termes, et pour reprendre une formule de Sophie Wahnich, la prison produit du " patrimoine négatif ${ }^{50}$ ». La notion de patrimoine négatif est discutée, tout autant peut-être que celle de patrimoine carcéral pour cette raison précise que le patrimoine carcéral est dans une large mesure un patrimoine négatif, surtout s'il entend prendre en charge «la mémoire des individus pour lesquels ces bâtiments ont été conçus ${ }^{51}$ » ou bien la conservation d'un instrument lié à la peine de mort.

Les statuts matériels et symboliques de ces deux objets diffèrent considérablement: mobilier imposant jusqu'à l'encombrement qui incarne le châtiment, écritures fragiles et éphémères produites par ceux qui transitent entre les murs des cellules. Pour la guillotine, il s'est agi d'inscrire au passé l'histoire de cet instrument, «le temps d'apaiser les esprits ", en raison de la charge polémique et affective dont il était porteur. La visibilité de cet objet est en tout point opposée à celle des graffitis. L'histoire de ces derniers est en effet en grande partie l'histoire d'un regard ou d'un non-regard posé sur des écritures au mieux invisibles, au pire indésirables. Ces deux cas de figure ont néanmoins en commun de susciter des réactions axiologiquement contrastées.

\section{NOTES}

1. Décret $n^{\circ}$ 2013-157 du 21 février 2013 portant création de l'établissement public du musée des civilisations de l'Europe et de la Méditerranée (Mucem).

2. CHEVALLIER Denis, "Collecter, exposer le contemporain au Mucem », Ethnologie française, vol. 38, $\mathrm{n}^{\circ} 4,2008$, p.631-637, disponible en ligne https://www.cairn.info/revue-ethnologiefrancaise-2008-4-page-631.htm [lien valide en novembre 2021].

3. CHEVALLIER Denis, «Collecter, exposer le contemporain au Mucem », Ethnologie française, p. 7.

4. L'une en bois de chêne verni noir (1982.50.1), l'autre rouge (1982.50.2).

5. SANCHEZ Jean-Lucien, "Histoire de la prison des Baumettes", Musée Criminocorpus, exposition organisée par le musée d'Histoire de la justice, des crimes et des peines le 12 août 2019, disponible en ligne https://criminocorpus.org/fr/expositions/anciennes/prisons/histoirede-la-prison-des-baumettes/ [lien valide en novembre 2021].

6. La prison a ainsi fait l'objet d'un rapport accablant en décembre 2012 par le contrôleur général des lieux de privation de liberté, constatant une violation grave des droits fondamentaux des personnes privées de liberté, à la suite duquel la décision a été prise de moderniser les bâtiments. Voir https://www.cglpl.fr/wp-content/uploads/2014/04/Rapport-de-visite-du-CP-deMarseille.pdf [lien valide en novembre 2021]. Cela s'est traduit par la construction du site Baumettes 2 et la destruction du bâtiment dit des «Baumettes historiques", appelé à être 
remplacé par un troisième bâtiment, Baumettes 3. Dans l'intervalle, la prison se trouve partiellement désaffectée, au cœur d'enjeux patrimoniaux paradoxaux. En effet, si les bâtiments sont promis à la destruction, les murs extérieurs de l'établissement, ornés de sept statues d'Antoine Sartorio (1885-1988) représentant les sept péchés capitaux, sont aujourd'hui classés.

7. MONJARET Anne, «À l'ombre des murs palimpsestes. Les graffiti carcéraux ou faire avec les aveux de l'histoire ", Gradhiva, $\mathrm{n}^{\circ} 24,2016$, p. 164-189, disponible en ligne https:// journals.openedition.org/gradhiva/3288 [lien valide en novembre 2021].

8. Bien qu'il s'agisse ici d'étudier la patrimonialisation de deux guillotines, l'étude concerne plus précisément celle en bois de chêne verni noir (1982.50.1) exposée au musée d'Orsay, au Mucem et au centre pénitentiaire des Baumettes.

9. Procès-verbal du comité consultatif des musées nationaux du 12 novembre 1981, dossier d'œuvre ${ }^{\circ}$ 1982.50.1, Mucem.

10. ATTALI Jacques, Verbatim. Chronique des années 1981-1986, Paris, Fayard, coll. « Documents, témoignage ", tome 1,1993-1995, p. 94 et Le Monde, 18 septembre 1981.

11. Procès-verbal du comité consultatif des Musées nationaux du 12 novembre 1981, dossier d'œuvre $n^{\circ}$ 1982.50.1, Mucem.

12. Nous n'avons pas trouvé ou pu consulter de document précisant cette demande de mise en dépôt.

13. Courrier du musée Carnavalet, N/Réf. 1714, 2 août 1983, dossier d'œuvre ${ }^{\circ}$ 1982.50.1, Mucem. 14. Courrier du musée national des Arts et Traditions populaires, N/Réf. AM/SV 11252, 10 août 1983, dossier d'œuvre ${ }^{\circ}$ 1982.50.1, Mucem.

15. BARON Marie-Guy, "Que sont devenus la guillotine et le bourreau », Le Figaro, 10 janvier 1985. Dossier d'œuvre Mucem.

16. Le Monde, 10 mai 1984.

17. BADINTER Robert, Les Épines et les roses, Paris, Fayard, coll. « Documents, témoignage », 2011, p. 229-231.

18. BARON Marie-Guy, "Que sont devenus la guillotine et le bourreau », Le Figaro, 10 janvier 1985. Dossier d'œuvre Mucem.

19. "Crime et châtiment » exposition organisée par le musée d'Orsay, (Paris, 16 mars-27 juin 2010), projet de Robert Badinter, commissariat général de Jean Clair. En savoir plus via https:// www.musee-orsay.fr/fr/expositions/crime-et-chatiment-196195 [lien valide en novembre 2021].

20. Bon d'enlèvement, dossier d'œuvre $\mathrm{n}^{\circ} 1982.50 .1$, Mucem.

21. Robert Badinter, intervention au Sénat, «Célébration du trentième anniversaire du vote par le Sénat de l'abolition de la peine de mort », séance exceptionnelle du 30 septembre 2011.

22. Voir POULAIN France, "Le château de Gaillon: un passé carcéral volontairement oublié. L'apport de l'archéologie des graffitis pour comprendre un site ", In Situ. Revue des patrimoines dans le prochain numéro consacré au patrimoine judiciaire à paraître courant 2022.

23. La notion de "patrimoine carcéral » a émergé dans un contexte singulier, celui du début des années 2000, lorsque l'administration a accéléré le grand chantier de modernisation et d'extension du parc carcéral, suite à la publication de plusieurs rapports accablants émanant d'ONG et des institutions européennes visant les conditions d'incarcération et l'état de salubrité des bâtiments. Sur la notion de «patrimoine carcéral », voir les travaux de Jean-Claude Vimont. Voir aussi SOPPELSA Caroline, «Architecture pénitentiaire. Mémoire historique : l'ambivalence des représentations ", Sociétés \& Représentations, vol. 30, n 2, 2010, p. 83-96, disponible en ligne https://www.cairn.info/revue-societes-et-representations-2010-2-page-83.htm [lien valide en novembre 2021].

24. C'est ce que souligne Jacqueline $Z$. Wilson dans son étude des prisons désaffectées australiennes transformées en musées : les graffitis sont régulièrement effacés avant ouverture au public. Voir WILSON Jacqueline Z., Prison. Cultural Memory and dark tourism, New York, Peter Lang, 2008, p. 68. 
25. Les graffitis ne semblent pas constituer une source d'information pour l'administration pénitentiaire par exemple, ou un motif réel de sanction. En tout état de cause, à l'époque contemporaine, les graffitis entrent dans cette zone de tolérance permise par l'administration pénitentiaire : interdits sur le papier, ils font néanmoins partie du paysage visuel de bâtiments parfois extrêmement vétustes, comme la prison des Baumettes. En ce sens, ils sont comparables aux objets fabriqués avec les moyens du bord par les détenus pour améliorer leur quotidien en détention : interdits mais tolérés par les gardiens.

26. FABRE Daniel (dir.), Écritures ordinaires, Paris, POL, coll. « Essais », 1993.

27. Ces dessins de bagnards faisaient partie des archives personnelles du commandant Cesari, ramenées à Nice vers 1862, et données au musée par la petite-fille du commandant en 2001. Préinventaire des archives du commandant Cesari, Mucem.

28. L'expression est de l'épigraphiste Armando Petrucci et concerne tous les écrits qui sont affichés dans l'espace public depuis les enseignes, devises au fronton des monuments, jusqu'aux écritures exposées déviantes comme les inscriptions et graffitis anonymes. Voir PETRUCCI Armando [1986], Jeux de lettres. Formes et usages de l'inscription en Italie, $11^{e}-20^{e}$ siècles, Paris, éditions de l'École des hautes études en sciences sociales, coll. « Recherches d'histoire sociale », 1993.

29. Il faut mentionner une initiative contemporaine similaire, celle du Mass-Observation en Angleterre, qui mène une campagne de collecte des graffitis pendant la Deuxième Guerre mondiale. Voir MAK Ariane, « Worktown. Les enquêtes fondatrices du Mass-Observation à Bolton (1937-1938) ", in HATZFELD Nicolas, VIGNA Xavier, LESPINET Isabelle \& GEERKENS Éric (dir.), Les Enquêtes ouvrières dans l'Europe contemporaine. Entre pratiques scientifiques et passions politiques, Paris, La Découverte, coll. « Recherches », 2019, p. 400-413.

30. CARLE Zoé, «Slogans et graffitis contestataires en Méditerranée : des voix aux murs », projet de recherche postdoctoral LabexMed-MUCEM, 2019-2020.

31. Voir ADELL Nicolas, "L'Anthropologie ou les promesses du crépuscule », L'Homme, $n^{\circ} 218$, 2016, p. 67-83, https://journals.openedition.org/lhomme/28927 [lien novembre 2021].

32. ARTIÈRES Philippe, Rêves d'histoire. Pour une histoire de l'ordinaire [2006], Paris, Verticales, coll. « Verticales », 2014, p. 14.

33. Voir l'exposition en ligne «La mémoire des murs» via https://criminocorpus.org/fr/ expositions/anciennes/art-et-justice/la-memoire-des-murs/ [lien valide en novembre 2021].

34. HEINICH Nathalie, La Fabrique du patrimoine: de la cathédrale à la petite cuillère, Paris, éditions de la Maison des sciences de l'homme, coll. «Ethnologie de la France », 2009, p. 89-119, disponible en ligne https://books.openedition.org/editionsmsh/2642 [lien valide en novembre 2021].

35. Voir, extrêmement complet : MONJARET Anne, « À l'ombre des murs palimpsestes. Les graffiti carcéraux ou faire avec les aveux de l'histoire ", Gradhiva.

36. MONJARET Anne, «À l'ombre des murs palimpsestes. Les graffiti carcéraux ou faire avec les aveux de l'histoire », Gradhiva.

37. FABRE Daniel (dir.), Écritures ordinaires.

38. De nombreux travaux, en ethnologie du patrimoine, histoire ou anthropologie de l'écriture ont pris ces écritures pour objet. On mentionnera l'initiative du Centre des monuments nationaux, sous la direction de Laure Pressac, signe de l'intérêt croissant que portent désormais les lieux patrimoniaux aux inscriptions « déviantes». Voir PRESSAC Laure (dir.), Sur les murs. Histoire(s) de graffitis, Paris, éditions du patrimoine, coll. « Hors collection », 2018.

39. En effet, il faut attendre les années 2000 pour qu'ils fassent l'objet d'un engouement certain et toujours plus prononcé. Voir la liste des travaux établie par MONJARET Anne, «À l'ombre des murs palimpsestes. Les graffiti carcéraux ou faire avec les aveux de l'histoire », Gradhiva, p. 15.

40. ARTIÈRES Philippe, Clinique de l'écriture. Une histoire du regard médical sur l'écriture [1997], Paris, La Découverte, coll. « La Découverte / Poche », 2013. 
41. FOUCAULT Michel, Surveiller et punir. Naissance de la prison, Paris, Gallimard, coll. « Bibliothèque des Histoires », 1975, p. 255.

42. ARTIÈRES Philippe, Clinique de l'écriture. Une histoire du regard médical sur l'écriture, p. 83. 43. Un phénomène comparable concerne les graffitis de hobos (vagabonds) américains, dont les inscriptions dans les wagons de fret, le long des chemins, perçues comme un code d'initiés issu de mondes sociaux marginaux, impossibles à pénétrer, ont fait l'objet d'une fascination singulière.

44. Voir RENNEVILLE Marc, «Le criminel-né : imposture ou réalité? " in «Histoire de la criminologie. Autour des Archives d'anthropologie criminelle 1886-1914 », Criminocorpus, 2005, [en ligne] http://journals.openedition.org/criminocorpus/127 [lien valide en novembre 2021]. Voir aussi «La criminologie perdue d'Alexandre Lacassagne (1843-1924)» in « Histoire de la criminologie. Autour des Archives d'anthropologie criminelle 1886-191 », Criminocorpus, 2005, [en ligne] http://journals.openedition.org/criminocorpus/112 [lien valide en novembre 2021].

45. CALET Henri, Les Murs de Fresnes, Paris, Aux éditions des quatre vents, 1945.

46. PITRÈ Giuseppe \& SCIASCIA Leonardo, Urla senza suono. Graffiti e disegni dei prigionieri dell'Inquisizione, Palerme, Sellerio, 1999.

47. FABRE Daniel, «I libri di pietra », Prima persona. Percorsi autobiografici, $\mathrm{n}^{\circ}$ 17, 2007, p. 49-55. Cité par MONJARET Anne, «À l'ombre des murs palimpsestes. Les graffiti carcéraux ou faire avec les aveux de l'histoire », Gradhiva.

48. VIMONT Jean-Claude, «Graffiti en péril?», Sociétés \& Représentations, $\mathrm{n}^{\circ} 25,2008$, p. 193-202, disponible en ligne https://www.cairn.info/revue-societes-et-representations-2008-1page-193.htm [lien valide en novembre 2021].

49. Fréquents sont les rapprochements avec un mouvement de fond en photographie et dans les pratiques urbaines ou péri-urbaines des jeunes comme le mouvement Urbex (Urban Exploration) qui prend pour objet un patrimoine industriel ou administratif laissé à l'abandon.

50. WAHNICH Sophie, "L'impossible patrimoine négatif », Les Cahiers Irice, vol. 7, $\mathrm{n}^{\circ}$ 1, 2011, p. 47-62, disponible en ligne https://www.cairn.info/revue-les-cahiers-irice-2011-1-page-47.htm [lien valide en novembre 2021]. Voir aussi de la même auteur «L'immigration produit du patrimoine négatif. Le rôle du musée », Communications, $n^{\circ}$ 100, 2017, introduction : « Des passés déplacés. Mémoires des migrations », p.119-135, disponible en ligne https://www.cairn.info/ revue-communications-2017-1-page-119.htm?contenu=article [lien valide en novembre 2021].

51. SOPPELSA Caroline, "Architecture pénitentiaire. Mémoire historique: l'ambivalence des représentations », Sociétés \& Représentations.

\section{RÉSUMÉS}

Le musée des civilisations de l'Europe et de la Méditerranée (Mucem) conserve un certain nombre d'éléments liés à l'univers carcéral et à ses représentations. La prison est présente dans les collections sous la forme d'estampes, de caricatures, de cartes postales, collectées au gré de différentes acquisitions et / ou enquêtes-collectes visant à rassembler et à documenter des ensembles d'objets tant matériels qu'immatériels, témoins de faits de société. Le musée a par ailleurs la spécificité d'abriter une collection consacrée au mouvement graff, constituée au début des années 2000. Il s'est engagé depuis juin 2019, en collaboration avec l'Inventaire général du patrimoine culturel PACA, dans une enquête-collecte au sein de la prison des Baumettes, prenant spécifiquement les graffitis carcéraux comme objet. 
Les Baumettes dites historiques ferment définitivement en juin 2018 avant une démolition prévue pour mars 2020, reportée à juillet 2021. Le projet "Adieu Baumettes ", imaginé par l'Administration pénitentiaire et inauguré pour les Journées Européennes du Patrimoine le 18 septembre 2019, souhaite rendre les Baumettes aux Marseillais en rouvrant quelques semaines la partie historique et en proposant des visites accompagnées d'une programmation culturelle largement consacrée à l'histoire de cette prison où a eu lieu, en 1977, la dernière exécution capitale avant l'abolition de la peine de mort. Dans ce cadre l'Administration pénitentiaire sollicite le prêt de la guillotine conservée dans les collections du Mucem. L'enquête-collecte "Graffitis et créations carcérales", est contemporaine à ce projet d'exposition réactivant un objet particulier des collections du musée. Déclenchée par la destruction prochaine des bâtiments, elle se donne comme mission la conservation d'urgence d'une mémoire du site qui passe notamment par une campagne photographique s'attachant à constituer un corpus autour des graffitis carcéraux en prolongement de la collection Graff et par une collecte d'objets venant compléter les collections déjà existantes sur la prison. L'extension vers les graffitis carcéraux entendus comme productions de justiciables tout autant dignes d'intérêt que les caricatures ou les dessins conservés par le Mucem fait écho à l'intérêt qui se manifeste à la fois dans le champ universitaire et au sein de l'Administration pénitentiaire pour ces objets ambigus dont le statut oscille entre témoignage, création et dégradation.

The "musée des civilisations de l'Europe et de la Méditerranée", the Museum of Europe and Mediterranean Civilisations (Mucem) preserves a number of elements related to the world of prison and its representations. Prison is part of the collections in the form of etchings, caricatures, postcards collected through the diverse acquisitions and / or survey-collections that seek to gather, and document sets of objects both tangible and intangible, which bear witness to social issues. The museum has the specific feature to hold a collection dedicated to the graffiti movement, built up in the early 2000s. It has been involved since June 2019, together with the "Inventaire général du patrimoine culturel PACA", PACA region's General inventory of heritage, in a survey-collection in the Baumettes prison, specifically focusing on prison graffiti.

The so-called historical Baumettes closed down permanently in June 2018 before its demolition scheduled for March 2020, postponed until July 2021. The "Adieu Baumettes" ("Farewell Baumettes") project, conceived by the penitentiary Administration and launched with the European Heritage Days on 18 September 2019, hoped to give the Baumettes back to the inhabitants of Marseilles by reopening for a few weeks the historical part and by offering tours together with a cultural programming largely devoted to the history of this prison, where the last capital punishment was carried out in 1977, before the abolition of the death penalty. Within this context, the penitentiary Administration requested the loan of the guillotine kept in the collections of the Mucem. The survey-collection on "Graffiti and prison creations", coincided with this exhibition project showcasing a specific object of the museum's collections. Triggered by the coming destruction of the buildings, the mission of this survey-collection was the emergency preservation of the site's memory which required in particular a photographic campaign that focused on the creation of a corpus about prison graffiti in line with the Graff collection and a collecting of objects to complete the existing collections on prison. The extension to prison graffiti seen as productions of convicts just as worthy of interest as caricatures or drawings preserved by the Mucem, reflects the growing interest, within both the academic field and the penitentiary Administration, in those ambiguous objects whose status oscillates between evidence of the past, creation and damage. 
INDEX

Mots-clés : graffiti carcéral, Baumettes, écritures exposées, guillotine

Keywords : prison graffiti, Baumettes, exposed writings, guillotine

\section{AUTEURS}

\section{ZOÉ CARLE}

Postdoctorante LabexMed-Mucem

zoe.carle@gmail.com

\section{JEAN-FABIEN PHILIPPY}

Chargé de mission auprès de la directrice scientifique et des collections, Mucem, Marseille jean-fabien.philippy@mucem.org 